\title{
Acupuncture, Incommensurability, and Conceptual Change ${ }^{1}$ \\ Paul Thagard and Jing Zhu \\ Philosophy Department \\ University of Waterloo \\ Waterloo, Ontario, N2L $3 G 1$ \\ pthagard@watarts.uwaterloo.ca
}

\begin{abstract}
This paper is an investigation of the degree of incommensurability between Western scientific medicine and traditional Chinese medicine, focusing on the practice and theory of acupuncture. We describe the structure of traditional Chinese medicine, oriented around such concepts as yin, yang, qi, and xing, and discuss how the conceptual and explanatory differences between Western medicine and traditional Chinese medicine generate impediments to their comparison and evaluation. We argue that the linguistic, conceptual, ontological, and explanatory impediments can to a large extent be overcome, and conclude that the dramatic differences between Western and traditional Chinese medicine do not provide insurmountable barriers to rational evaluation of acupuncture. We conclude with a discussion of the intentional and emotional aspects of conceptual change.
\end{abstract}

\section{Introduction}

In November 1997, the U.S. National Institutes of Health conducted a consensus development conference on acupuncture. Like the previous 106 consensus conferences sponsored by NIH since 1977, the acupuncture conference consisted of presentations to a panel charged with making recommendations concerning medical practice. But it was unusual in being the first co-sponsored by the new NIH Office of Alternative Medicine, and the first to consider therapies from outside the Western medical tradition. Acupuncture, involving the insertion of needles under the skin at prescribed positions, is a central component of the two-thousand-and-five-hundred-year old system of traditional Chinese medicine. This system of medicine employs a conceptual system very different from the one that has evolved in Europe and America over the past century and a half. 
Allchin (1996, p. S107) says that the contrasting traditional Chinese and Western views of acupuncture "offer a particularly deep version of Kuhnian incommensurability."

In the 1960s, philosophy of science was scandalized by the suggestion that competing theories might be incommensurable with each other (Kuhn 1970; Feyerabend 1981). If two conceptual systems such as those comprising the oxygen and phlogiston theories of combustion are radically different, then rational comparison and assessment of them becomes difficult if not impossible. Subsequent discussions have shown that claims of radical incommensurability in the history of science were greatly exaggerated (see, for example, Laudan 1984, Nersessian 1984, Thagard 1992). Although competing scientific theories may indeed occupy very different conceptual systems, there is usually enough conceptual, linguistic, and evidential overlap that rational assessment can take place. In Kuhn's most recent writings, incommensurability is no longer a dramatic impediment to the comparability of theories, but rather an unthreatening observation on difficulties of translation and communication (Kuhn 1993).

Nevertheless, even if rationality-destroying incommensurability is rare within science, it would not be surprising if it occurred at the boundaries between science and nonscience. Contrast, for example, the cosmology of modern astrophysics with that of Australian aboriginals. The ontology, concepts, and linguistic context of these cosmologies are so radically different that explicit comparison and rational evaluation in terms of the standards of each will be very difficult. Of course, from the empirical and theoretical perspective of Western science, the superiority of astrophysics over aboriginal cosmology is obvious, as are the advantages of the scientific perspective. Still, it is possible that radical incommensurability, of the sort that Kuhn and Feyerabend mistakenly attributed within Western science, may arise between science and alternative views of the world.

This paper is an investigation of the degree of incommensurability between Western scientific medicine and traditional Chinese medicine, focusing on the practice 
and theory of acupuncture. In the next section, we very briefly sketch the conceptual and explanatory structure of modern medicine. We then provide a more detailed description of the structure of traditional Chinese medicine, oriented around such concepts as yin, yang, qi, and xing. It is then possible to discuss how the conceptual and explanatory differences between Western medicine and traditional Chinese medicine generate impediments to their comparison and evaluation. We outline linguistic, conceptual, ontological, and explanatory difficulties that might be taken to imply that traditional ideas about acupuncture are incommensurable with Western medicine. We argue, however, that the difficulties can to a large extent be overcome, as they were at the NIH meeting in the service of the attempt to improve medical treatments. Our conclusion is that the dramatic differences between Western and traditional Chinese medicine do not provide insurmountable barriers to rational evaluation of acupuncture. The main positive contribution of this paper is that its display of the conceptual and explanatory structure of traditional Chinese medicine provides an informative contrast that highlights aspects of the nature of Western science. We conclude with a discussion of how appreciation of different medical frameworks can require conceptual change that is both intentional and emotional.

Our discussion will distinguish between strong and weak incommensurability. Two theories or conceptual schemes are strongly incommensurable if they are mutually unintelligible, so that someone operating within one conceptual scheme is incapable of comprehending the other. Weak incommensurability, however, does not imply mutual unintelligibility, but only that the two conceptual schemes cannot be translated into each other. If traditional Chinese medicine were strongly incommensurable with Western medicine, there would be no possibility of rational evaluation of Chinese medicine from the Western perspective. We shall argue, however that the weak incommensurability that holds between the two medical traditions does not prevent rational evaluation of practices such as acupuncture. 


\section{Western Scientific Medicine}

What is the structure of modern medicine? Biomedical theories are not naturally represented as formal axiom systems, but can naturally be characterized in terms of hierarchical cognitive structures (Schaffner 1993; Thagard 1996, 1999). Figure 1 depicts at a very general level the conceptual and explanatory structure of scientific medicine as it has evolved since Pasteur proposed the germ theory of disease in the 1860s. Diseases can be classified according to the bodily systems that they affect, for example into heart or skin diseases, but a deeper classification is based on the causes of disease. Modern medicine recognizes four kinds of causes of disease: infectious agents such as viruses, nutritional deficiencies such as lack of vitamin $\mathrm{C}$, molecular-genetic disorders such as cancer, and autoimmune reactions such as the attack on the connective tissue that produces lupus erythematosus.

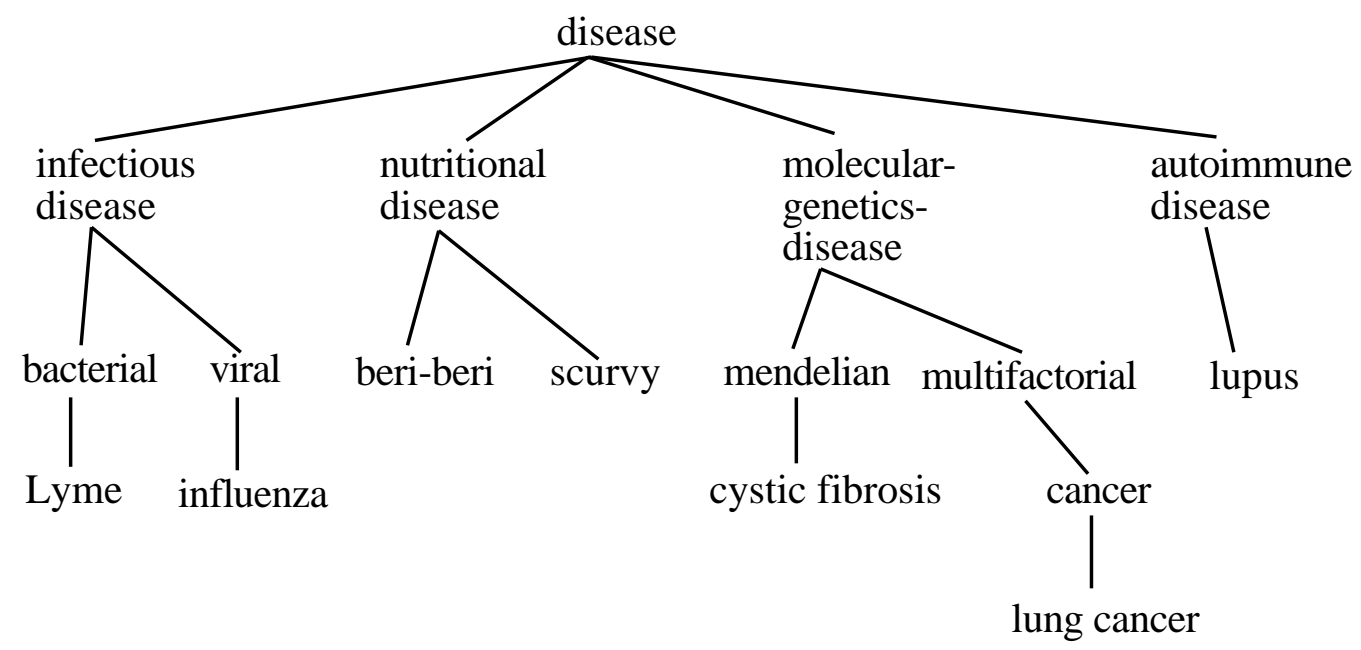

Figure 1. Hierarchical organization of disease explanations, with examples of particular diseases. From Thagard (1999).

For each class of disease, there is an explanation schema that specifies a typical pattern of causal interaction. ${ }^{2}$ Infectious diseases fall under the following explanation schema that became very successful in the nineteenth century:

Germ Theory Explanation Schema:

Explanation target: 
Why does a patient have a disease with symptoms such as fever?

\section{Explanatory pattern:}

The patient has been infected by a microbe.

The microbe produces the disease and symptoms.

To apply this schema to a particular disease, we need to replace the terms in boldface with specific examples or classes of examples. For example, influenza instantiates the schema by specification of symptoms such as fever, aches, and cough and by specification of the class of flu viruses that cause the symptoms. Figure 2 diagrams the causal structure of the germ theory of disease. Germs such as viruses and bacteria cause infections that produce symptoms that develop over time, constituting the course of the disease. Treatments such as antibiotics and vaccines can kill the germs or inhibit their growth, thereby stopping or preventing the infection that produces the symptoms.

Analogous explanation schemas for nutritional, molecular-genetic, and autoimmune diseases have been presented elsewhere (Thagard 1996, 1999). Here, we have only presented enough of the conceptual and explanatory structure of modern medicine to provide a contrast with an alternative approach.

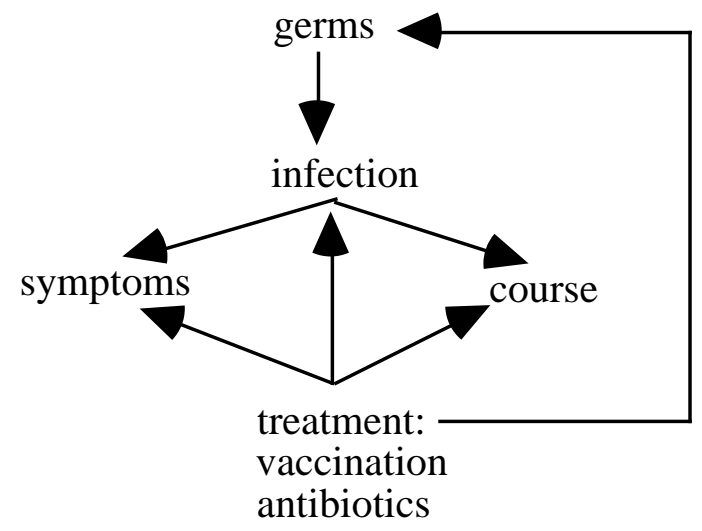

Figure 2. Causal structure of the germ theory of disease. From Thagard (1999).

\section{Traditional Chinese Medicine}


Traditional Chinese medicine developed for more than 2,500 years almost entirely free from Western influences. Its theories and practices of diagnosis and treatment are remarkably different from those of Western medicine. Some of its treatments such as herbal therapies and acupuncture are currently receiving increasing attention in the West, part of a rapidly growing interest in alternative and complementary medicine. As section 5 will describe, numerous experimental and clinical studies have confirmed that acupuncture treatments can relieve pain and reduce nausea. However, the theories of traditional Chinese medicine seem bizarre from the point of view of modern Western medicine. Even if some proponents of traditional Chinese medicine claim that Western and Chinese medicine should complement each other, they admit that Chinese medicine is organized on totally different principles (Porkert and Ullmann 1988, p. 55). In this section, we will try to display this organization and outline the conceptual and explanatory structure of traditional Chinese medicine.

\section{The Balance of Yin and Yang}

In ancient China, people believed that everything in the universe consists of two opposite but complementary aspects or forces, which combine to create a whole unit (Zhen 1997). Yin and yang refer to the two basic categories of the universe, negative and positive respectively, and they are in constant flux. Every thing or event in the world is to be regarded as the interaction of an active and a conservative force, each of which has its own peculiar characteristics that determine the nature of the thing or event. According to Yellow Emperor's Classic of Internal Medicine: Plain Questions (chapter 5), one of the most important and original classics of traditional Chinese medicine, "the principle of yin and yang is the way by which heaven and earth run, the rule that everything subscribes, the parents of change, the source and start of life and death" (Guo, 1992).

The original meaning of the two words yin and yang in Chinese referred, respectively, to the side of a mountain that lies in shadow and the side that lies in sun. Yin could also refer to the shaded bank of a river, yang to the sunlit bank. But the terms 
are no longer strictly confined to their original meaning and have become basic and abstract categories in both Chinese philosophy and people's ordinary thinking. Typically, dynamic, positive, bright, warm, solid features are defined as yang, while static, negative, dark, cold, liquid and inhibiting features are characterized as yin. Sunlight and fire are hot while moonlight and water are cool, so that the sun and fire are yang while the moon and water are yin. Yin and yang are complementary to and interdependent on each other, even though they are opposites. For every individual thing, the yin and yang it contains do not remain in a static state, but are constantly in a dynamic equilibrium affected by the changing environment.

Like everything else, the human body and its functions are all governed by the principle of yin and yang. Remaining healthy and functioning properly require keeping the balance between the yin and yang in the body. Diseases arise when there is inequilibrium of yin and yang inside the body. This principle is central to traditional Chinese medicine, and its application dominates the diagnosis, treatment and explanation of diseases. For example, a patient's high fever, restlessness, a flushed face, dry lips and a rapid pulse are yang symptoms. The diagnosis will be a yin deficiency, or imbalance brought by an excess of yang over yin. Once the yin-yang character of a disease is assessed, treatment can restore the balance of yin and yang, for example by using yin-natured herbs to dampen and dissipate the internal heat and other yang symptoms. The imbalance of yin and yang can be caused by either exogenous factors, such as climate, traumatic injuries and parasites, or endogenous factors, such as extreme emotional changes (anger, melancholy, anxiety, and so on), abnormal diet, intemperance in sexual activities and fatigue. Figure 3 displays the structure of the causal network underlying the ying-yang explanation of disease. As with the germ theory of disease shown in figure 2 , causes produce a set of symptoms and their course of development 


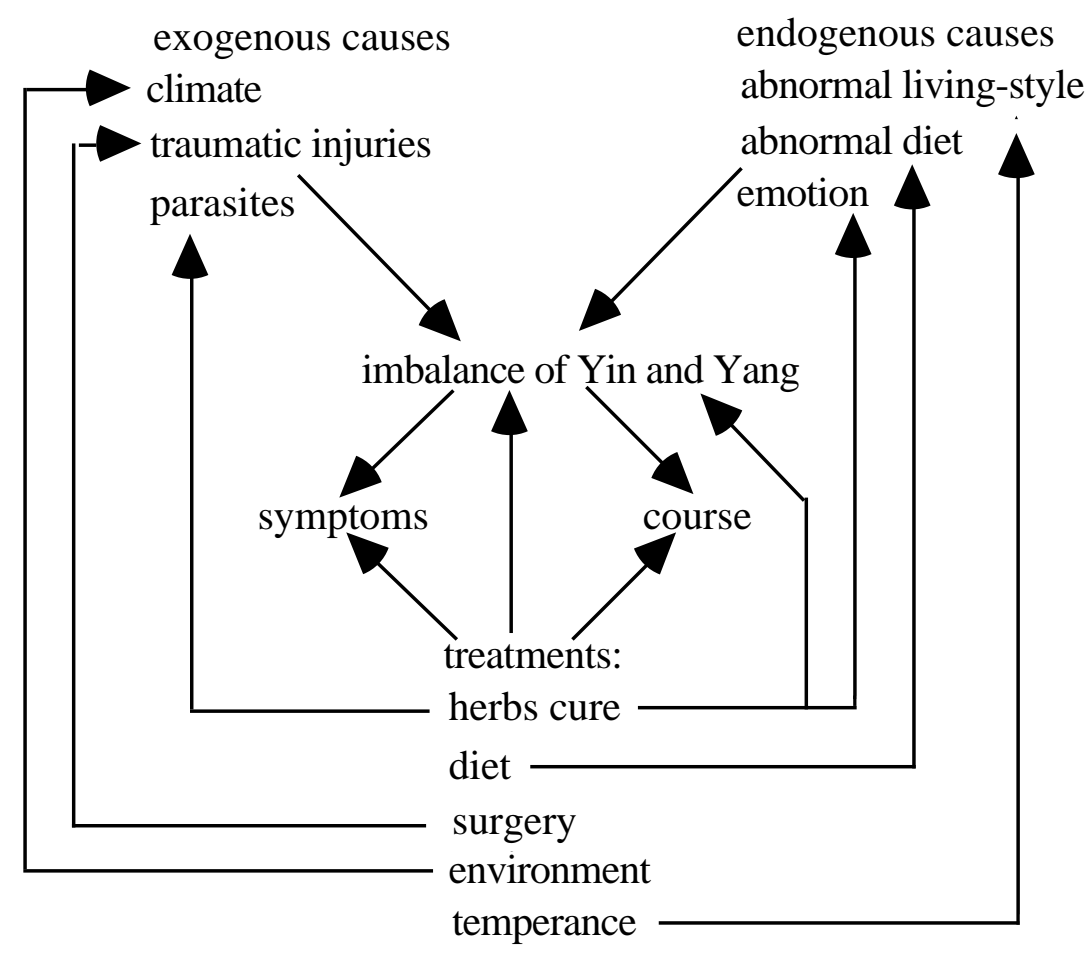

Figure 3. Causal structure of disease concepts in the theory of yin and yang balance.

The way in which this causal structure explains disease can also be described by the following schema:

Yin and Yang Balance Theory Explanation Schema:

Explanation target:

Why does a patient have a disease with associated symptoms? Explanatory pattern:

The patient's body is subject to exogenous and endogenous factors.

The factors produce an imbalance of yin and yang.

The imbalance of yin and yang produces the disease and symptoms.

This is the most general and fundamental pattern of disease explanation in traditional Chinese medicine, but there are also some more specific explanation schemas. 


\section{The Theory of the Five Xing}

According to Aristotle and Hippocrates, everything in the world consists of four fundamental elements: earth, air, fire, and water. Similarly, the ancient Chinese considered metal, wood, water, fire, and earth to be fundamental. In Chinese, each of these is a xing, and the five collectively are called five-xing. There are two important differences between the theory of five-xing and the theory of four elements in ancient Greece. First, even though the five xings were considered as the basic components of the universe, the ancient Chinese did not use them to analyze the substantial constitution of particular things. Rather, the five xings are five basic categories that can be used to classify things according to their properties and relationships to other things. Second, the five xings are not independent each other, but have significant relationships and laws of transformation among them. Hence instead of translating xing as "element", various commentators prefer to call five xings the "Five Transformation Phases" or the "Five Phases of Change" (Porkert and Ullmann 1988; Unschuld 1985).

There are two basic kinds of relation or sequence among five xings: Mutual Promotion (Production) and Mutual Subjugation (Conquest). The principle of Mutual Promotion says that five xings may activate, generate and support each other. It is through these promotions of the elements that five xings continue to survive, regenerate and transform. The sequence of Mutual Promotion is as follows: wood promotes fire, fire promotes earth, earth promotes metal, metal promotes water, water promotes wood, and wood again promotes fire. The principle of Mutual Subjugation, concerns relations such as restraining, controlling and overcoming. Mutual restraint keeps the balance and harmony among the five xings. Wood subdues earth; earth subdues water; water subdues fire; fire subdues metal; metal subdues wood; wood in its turn acts on earth. Figure 4 shows the Mutual Promotion and Mutual Subjugation relationship among five xings. 

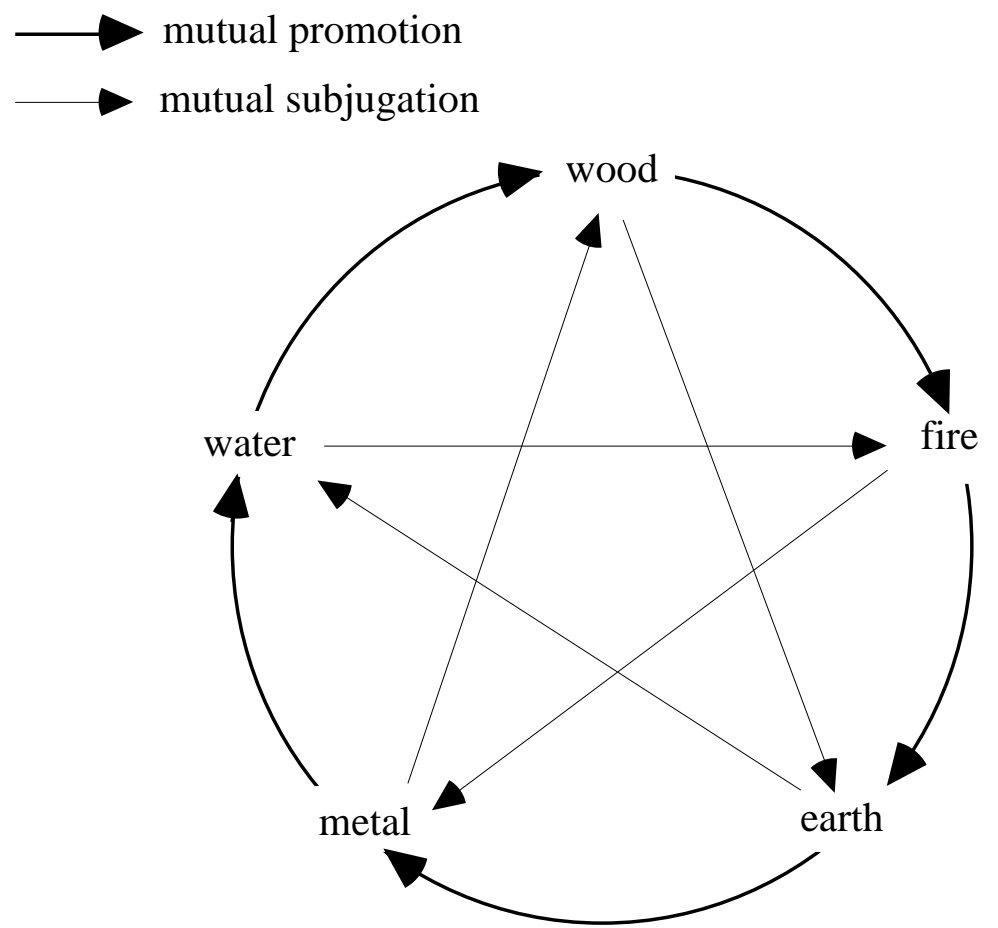

Figure 4. Mutual Promotion and Mutual Subjugation relations among five xings Adapted from Shen and Chen (1994), p. 17.

The meaning of the principles comes from experience. Fire is created when wood is burned. Ash (earth) is left after burning. All metals come from earth and liquefy on heating, while water is indispensable for growing trees and vegetation. These relations support the principle of Mutual Promotion (Production). On the other hand, the ancient Chinese noticed that trees grow on earth, impoverishing the soil. To prevent floods, dams and channels are built with earth. Water puts out fire while metals can be softened and melted by fire. A sword or ax made of metal can be used to fall a tree. These relations are summarized in the principle of Mutual Subjugation (Conquest).

Most things in the world can be classified into one of the five basic categories according to their properties, function and relations with others. Table 1 shows some objects relevant to diagnosis and treatment in traditional Chinese medicine. For example, the liver is similar to wood with respect to its mild features, and the heart warms the whole body so it is analogous to fire. The spleen is responsible for assimilation of 
nutrients and corresponds to the earth. The lung is clear, analogous to metal. The kidney is similar to water by virtue of its responsibility of regulating fluids in the body.

\begin{tabular}{|l|l|l|l|l|l|}
\hline Five Xings & \multicolumn{1}{|c|}{ Wood } & \multicolumn{1}{|c|}{ Fire } & \multicolumn{1}{|c|}{ Earth } & \multicolumn{1}{|c|}{ Metal } & \multicolumn{1}{|c|}{ Water } \\
\hline Five directions & East & South & Middle & West & North \\
\hline Five seasons & Spring & Summer & Late summer & Fall & Winter \\
\hline Five climates & Wind & Hot & Dampness & Dryness & Cold \\
\hline Five colors & Green & Red & Yellow & White & Black \\
\hline Five tastes & Sour & Bitter & Sweet & Pungent & Salty \\
\hline Five organs & Liver & Heart & Spleen & Lung & Kidney \\
\hline Five sense organs & Eye & Tongue & Mouth & Nose & Ear \\
\hline Five tissues & Tendon & Vessel & Muscle & Skin \& Hair & Bone \\
\hline Five emotions & Anger & Joy & Meditation & Grief & Fear \\
\hline
\end{tabular}

Table 1. The five categories of things classified according to the theory of

five-xing. Adapted from Shen and Chen (1994), p. 19.

In diagnosis and treatment, those things classified into the same kind are related to each other and have the same mutual relations with the objects in the neighboring categories. For example, a disease in the liver calls attention to the eyes, tendons, and the emotion of anger. Great anger is considered very harmful to the liver in traditional Chinese medicine. The liver pertains to wood, which flourishes in spring, so that liver diseases are prevalent in spring. The classification and correspondence in terms of five xings illustrate the mutual relationship between the human body, the seasons, climate factors, senses, and emotions. According to the principles of Mutual Promotion and Subjugation, the disease in one organ is not isolated from the other organs. A disease in liver (wood) is probably due to the functional deficiency of kidney (water), so the treatment should not only be aimed to the liver, but also enhance the function of kidney as well as those of others. Thus the perspective of traditional Chinese medicine is more 
holistic than the Western perspective, which tends to look for the seat of a disease in a particular organ.

The theory of five xings specifies aspects of the more general theory of yin and yang balance. The improper function of an organ is originally caused by imbalance of yin and yang, and in turn influences the harmony between other organs, which can also be analyzed in relation to yin and yang. Here is the explanatory schema:

Five Xings Explanation Schema:

Explanation target:

Why does a patient get a disease with associated symptoms?

\section{Explanation pattern:}

The imbalance of yin and yang causes one or more organs, which belong to the corresponding $\boldsymbol{x i n g}$, to malfunction.

The malfunction of one organ produces the disorder among all the organs, which are related between each other according to the rules of the theory of five xings.

The disorder among organs produces the disease and symptoms.

\section{The Circulation of $Q i$}

Another fundamental concept in traditional Chinese medicine is $q i$, which plays a central role in the theoretical background to such therapies as acupuncture, moxibustion, and massage. In the ordinary Chinese language, the term qi refers mostly to air or gas, and sometimes is also used to indicate a kind of emotion - anger. In the terminology of Chinese medicine, $q i$ has a different meaning. First, $q i$ is not a type of substance and has no fixed shape or constitution. Second, it is indispensable for life. Third, it is responsible for the resources of the function and operation of organs and the whole body. $Q i$ has variously been interpreted in terms of the Greek pneuma, vital force, or energy (Lloyd 1996, Lu and Needham 1980). Qi cannot be observed directly, but with long and assiduous training and practice, a doctor can supposedly detect its flow and changes in a 
patient. A person can also detect the flow of $q i$ and control its direction in some degree by exercises and meditation (Moyers 1993).

There are basically two kinds of qi, congenital qi inherited from one's parents and vital for one's life, and the other type acquired after birth. We get the acquired qi from food and water, which is assimilated by the spleen and stomach, and from the air inhaled by the lungs. The acquired $q i$ is constantly replenished, and is fundamental to maintaining the life activities of the body. Because $q i$ is dynamic, active, and warms the body, it falls under the yang category. Blood and body fluids, two kinds of fluids circulating inside the body, have the functions of nourishing and moistening. Therefore they belong to the yin category. $Q i$ is capable of producing and controlling blood, warming and nourishing the tissues, and activating the functions of organs.

Qi circulates along channels within the body called meridians. The system of meridians is unique to traditional Chinese medicine regarding human body, and does not correspond to blood vessels or nerves. The resource of $q i$ inside meridians comes from the internal organs, such as heart, spleen, lung, and stomach. As a unit, the system works to reinforce the coordination and balance of bodily functions.

Disease occur when the circulation of $q i$ is obstructed. Doctors need to identify where and why the flow of $q i$ is blocked and carry out the proper treatment to restore the circulation of $q i$. Deficiency of $q i$ can also cause illness, and the appropriate treatment is to replenish it. Figure 5 displays the causal structure of the theory of $q i$, and we can summarize disease explanations in the following schema:

Theory of Qi Explanation Schema:

Explanation target:

Why does a patient have a disease with associated symptoms?

Explanation pattern:

The body of the patient contains a meridian system which conducts the flow of qi. 
An obstruction occurs that blocks the flow of $q i$.

The $q i$ blockage produces the disease and symptoms.

disorder or malfunction of organs

disharmony of the interaction between $q i$ and blood

weakness, fatigue, emotion...

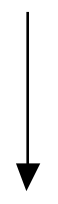

deficiency or obstruction circulation of $q i$

in some locations of meridians

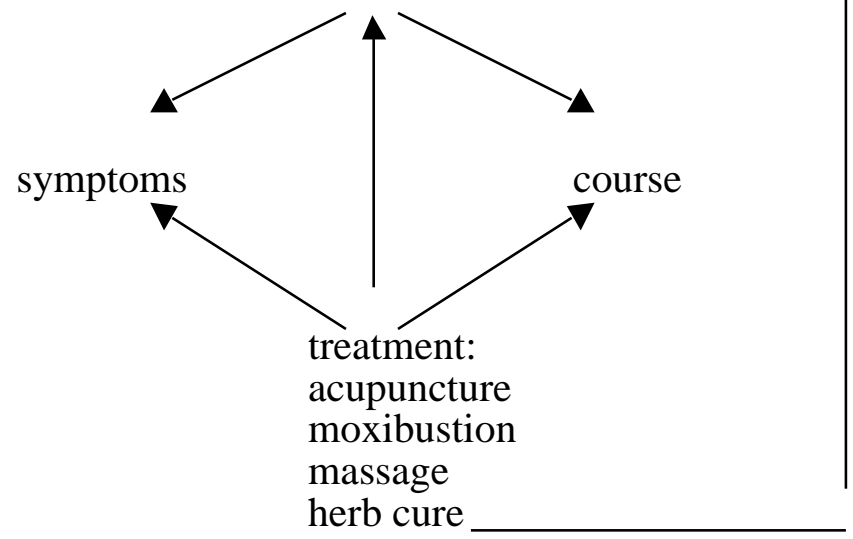

Figure 5. Causal structure of diseases due to $q i$ blockage.

Before proceeding to discuss philosophical issues concerning the relation of Western and Chinese medicine, we should stress that both these traditions are concerned primarily with the treatment of patients, and that the development of explanatory theories of diseases has been driven largely by this practical aim.

\section{Incommensurability}

It is obvious from our brief review that the conceptual and explanatory structure of traditional Chinese medicine is very different from that of Western medicine, but are these differences so large that they cannot rationally be compared? This question is of considerable current practical importance, because of live controversies concerning the medical legitimacy of acupuncture and other traditional Chinese treatments. If traditional Chinese medicine and Western medicine are mutually unintelligible, then evaluation of one within the framework of the other would seem to be impossible. We 
will now consider four potential impediments to mutual intelligibility: the linguistic differences between Chinese and Western languages, the differences in conceptual organization between Chinese and Western systems of thought, ontological differences between Chinese and Western theories, and explanatory differences involving notions of causality and correspondence.

\section{Linguistic Differences}

In both its spoken and written structure, the Chinese language is very different from European languages such as English, French, and German. Crucial terms from traditional Chinese medicine are not merely technical terminology, but are embedded in much broader linguistic usage. There are no terms in European languages that correspond even roughly to yin and yang, which are accordingly left untranslated. The term $q i$ q i i is often translated as "energy", but this translation is misleading if it generates an association with Western scientific concepts of electrical or mechanical energy, rather than with concepts such as breath, emotion, and force. Similarly, the translation of xing as "element" both adds and subtracts from the meaning of the Chinese term, since it adds the association of element as a fundamental constituent of the world, and loses the relational aspects of five-xing which are crucial to their explanatory roles.

The difficulty of translating Chinese medical terminology into European languages does not, however, show that Chinese and Western medicine are incommensurable. Even though there is no simple mapping of terms like ying, yang, xing, and $q i$ into English, the fact that the linguistic divergence can be systematically described by writers such as Lloyd (1996), Porker (1988), and Unschuld (1985) shows that comparison can proceed despite complexities of translation.

It is possible, however, that difficulties of translation run deeper than lack of corresponding terms for yin, yang, and so on. Bloom (1981) argues that the structure of the Chinese language is radically different from European languages in that it lacks distinct markings for counterfactual conditionals, so that it is not possible to make in 
Chinese such utterances as "If the Chinese government were to pass a law requiring that all citizens make reports of their activities to the police hourly, then what would happen?" He claims that Chinese speakers tend to brand the counterfactual as in some sense "unChinese". Bloom's claim is an instantiation of the linguistic relativity hypothesis of Whorf (1956), according to which differences in language generate radically different patterns of thought. If Bloom is right that the Chinese language enforces a non-Western attitude toward counterfactual conditionals, this may signal differences in understanding of causality, since causation involves counterfactual dependence between events (Lewis 1986). It is possible, therefore, that the explanatory claims of Chinese medicine are untranslatable into European languages because they presuppose a very different conception of causality.

Bloom's linguistic claims have, however, been strongly challenged. Cheng (1985) argued that the psychological experiments that Bloom used to support his claim of linguistic divergence were methodologically flawed and used poorly translated materials; and his experiments did not replicate (Au 1983). The Chinese language does in fact allow the statement of counterfactual conditionals, so there is no evidence of linguistic differences in the understanding of causality between Chinese and Western culture. Native speakers of Chinese, including the second author of this paper, report that Chinese people can understand counterfactuals very well. In sum, the substantial linguistic differences between the Chinese and European languages do not generate insurmountable barriers to comprehension and translation, and therefore do not support claims of incommensurability between Chinese and Western medicine.

\section{Conceptual Differences}

The problem of comparing Western and Chinese medicine is not just that the terms are different, but that the concepts are different in their places in conceptual hierarchical organization. Much current work in cognitive science views concepts as being organized in terms of kind hierarchies and part hierarchies (Thagard 1992). For 
example, a chicken is a kind of bird which is a kind of animal, and a beak is a part of a bird. There are problems, however, in placing Chinese concepts into the kind hierarchy of the Western system. Yin and yang do not seem to be kinds of anything familiar to Western thought. They are not things or substances or events or processes, and involve a kind of abstraction not found in Western concepts. So the problem of translating Chinese terms for them into English is not just a matter of finding the right term, but also reflects the fact that they do not fit into the Western conceptual organization.

Similar problems arise with xing and qi. The standard translation "elements" suggests that five-xing are a kind of thing or substance, but this classification fits poorly with their crucial relations of promotion and subjugation. $Q i$ would seem to be a kind of process, like energy, but its association with breath and force suggest that it is not a kind of process familiar to Western science or common sense. Thus the terms yin, yang, xing, and $q i$ all represent concepts that do not fit naturally in the Western hierarchy of kinds. Moreover, the kind hierarchy for diseases in Chinese medicine tends to divide them into ones caused by too much yin, too much yang, or by blockage of qi, rather than according to the Western classification in terms of infectious, nutritional, moleculargenetic, and autoimmune causes. Thus the differences between Western and Chinese medicine are conceptual (mental) as well as linguistic (verbal).

Additional differences are found in the part-hierarchies of the two systems. Traditional Chinese medicine did not permit autopsies and dissections, and so did not develop the detailed system of anatomy and physiology that evolved in Europe after the sixteenth century. According to Shen and Chen (1994), the term zang-fu refers to the five solid zang organs of the human body (heart, liver, spleen, lung, and kidney) and the six hollow viscera (gallbladder, stomach, small intestine, large intestine, bladder, and the sanjiao). The latter does not correspond to any part recognized in Western medicine, but consists of portions of the chest and abdomen that are thought to be important for the flow of $q i$. The function of other organs is sometimes described in ways similar to 
Western medicine, but is sometimes radically different; for example, the heart houses the mental faculties. Traditional Chinese medicine ignores some organs such as the pancreas that are viewed as medically important in Western medicine.

Although the kind hierarchies and part hierarchies of traditional Chinese medicine and Western medicine are obviously different, it would be an exaggeration to say that they are mutually unintelligible. Concepts such as $q i$ and sanjiao are undoubtedly alien to Western medicine, but their meaning can be acquired contextually from works such as Shen and Chen (1994). Conversely, practitioners of traditional Chinese medicine can acquire Western concepts such as germ, virus, and pancreas.

Buchwald and Smith (1997, p. 374) present a precise characterization of incommensurability that they report as Kuhn's final thoughts on the subject:

If two scientific schemes are commensurable, then their lexical structures can be fit together on one of the following two ways: (1) every kind, taxonomic or artefactual, in the one can be directly translated into a kind in the other, which means that the whole of one structure is isomorphic to some portion of the other; or (2) one structure can be grafted directly onto the other without otherwise disturbing the latter's existing relations. In the first case one scheme is subsumed by the other. In the second, a new scheme is formed out of the previous two, but it preserves intact all of the earlier relations among kinds. If neither case holds, the two systems are incommensurable.

Our discussion so far makes it clear that traditional Chinese medicine does not fit with Western medicine in the first way, since there is no direct translation of yin, yang, and kinds of Chinese disease into Western terminology. The second way of fitting does not work either, because grafting the two schemes together would require diseases to be classified simultaneously in conflicting ways, for example as both infectious and caused by excessive yin. Thus on the characterization of Buchwald and Smith, Chinese and 
Western medicine are incommensurable, although the lack of fit does not imply that they are not comparable or mutually intelligible. This is weak incommensurability arising from untranslatability, and contrasts with the strong incommensurability discussed by Laudan (1990, p. 121), who says that two bodies of discourse are incommensurable if the assertions made in one body are unintelligible to those utilizing the other.

\section{Ontological Differences}

The meaning of a concept is a matter of both its relation to other concepts and its relation to the world. So far, we have been discussing linguistic and conceptual differences between Western and traditional Chinese medicine, but it is also clear that the two approaches make very different claims about the world. Not only are yin, yang, qi, and five-xing not part of the ontology of Western science, they are not even kinds of entities, properties, or processes that are part of that ontology. Conversely, traditional Chinese medicine does not even consider many of the ontological claims of Western science, for example concerning such entities as disease-causing microbes. A Kuhnian would be tempted to say that Western physicians and traditional Chinese doctors live in different worlds.

There is, however, considerable overlap in the two ontologies. Both Western and traditional Chinese physicians examine peoples' bodies with similar perceptual systems, even if there are differences in some examination techniques. Pulse taking is different in the two cultures, in that traditional Chinese doctors aim to detect pulses with three different grades of force, but both Chinese and Western doctors grasp wrists and detect pulses. Despite their different ontological beliefs, it would be an exaggeration to place the traditional Chinese and Western doctors in different worlds.

\section{Explanatory Differences}

In Western scientific medicine, explanations are based on causal relations. A disease explains symptoms because the disease causes the symptoms, and the treatment is only judged to be effective if the treatment causes the elimination of disease. Although 
much of Western medicine is still based on the clinical experience of physicians rather than on scientific experiments, there is increased pressure to evaluate treatments using randomized, blinded, controlled trials (Sackett et al., 1996). Carefully controlled experiments are needed to determine whether treatments are causally effective, because they rule out alternative causes such as expectations and biases in physicians and patients (Thagard 1998).

According to Lloyd (1996, p. 113), traditional Chinese medicine similarly is interested in identifying causal factors, but it also has an additional explanatory style based on "correspondences". Unschuld (1994, p. 52) describes the role in ancient Chinese thought of concepts of magic correspondence and systematic correspondence, both of which are based on the principle that the phenomena of the visible and the invisible world stand in mutual dependence. Concepts like yin, yang, and $q i$ are embedded in a system of correspondences which involve non-causal dependencies. For example, the movement of $q i$ in the body is understand in part on the basis of the body having an upper part (yang) and a lower half (yin), and a left side (yang) and a right side (yin) (Unschuld 1994, p. 88).

Thus traditional Chinese medicine is closer to pre-scientific assumptions of homeopathic magic, which employs the principle that like corresponds to like, than it is to modern conceptions of causality. Thagard (1988, ch. 9) describes how much prescientific and pseudoscientific thinking is based on resemblance rather than causality. The causal mode of explanation found in current scientific medicine has no room for explanations based on resemblance and mystical correspondences, so it is difficult to compare the two kinds of explanation head to head. Here the debate between traditional Chinese medicine and Western science has to move to a metalevel involving the efficacy of the different styles of explanation. Evaluating traditional Chinese herbal medicine is also very difficult from the perspective of Western, evidence-based medicine, because prescribed herbal remedies often involve mixtures of numerous kinds of herbs suggested 
by correspondence-based ideas. Determining the causal effect of a single herb would be viewed as pointless within traditional Chinese medicine.

Even here, however, there is not complete breakdown of intelligibility, since traditional Chinese medicine does want to claim causal effectiveness for its treatments. Although it seems mysterious from the Western medical perspective why acupuncture places needles at certain points in the body that are thought to have the relevant correspondences, it is still possible to ask the question, common to both traditions, of whether the needling is causally effective. Hence the explanatory gap between traditional Chinese medicine and Western science is not so great as the gap, say, between Western science and fundamentalist religion, which claims that the primary source of evidence is a sacred text. Moreover, the gap between Western and Chinese medicine has shrunk over the centuries, in that the current explanatory role of systematic correspondences in Chinese medicine is much smaller than it was originally.

In sum, our discussion of the linguistic, conceptual, ontological, and explanatory differences between traditional Chinese medicine and traditional Western medicine has shown that the two approaches are not strongly incommensurable. Considerable mutual comprehension is possible, although it does not go so far as to permit translation of one conceptual system into the other; hence the two stems are weakly incommensurable. Let us now see how weak incommensurability affects the evaluation of acupuncture.

\section{Evaluating Acupuncture}

In the last section, we saw that the linguistic, conceptual, and ontological differences between traditional Chinese medicine and Western medicine do not constitute insuperable barriers to their rational comparison, although the explanatory differences are more serious impediments. A Western researcher demanding that acupuncture and other therapeutic practices be evaluated with respect to their explanatory coherence as shown by randomized and blinded clinical trials would be stymied by a proponent of traditional Chinese medicine who said that all this was simply irrelevant. But traditional 
Chinese medicine is not a mystical religion; it is aimed at improving people's health, and its practitioners sincerely believe that it succeeds. Hence even for the most orthodox practitioners of traditional Chinese medicine there is an empirical standard, not just a doctrinal one.

In a head-to-head clash between Western and traditional Chinese medicine, it would be necessary to choose one of the conceptual-explanatory systems as superior and reject the other. A skeptical Western physician, for example, could argue that Western medicine has incontrovertible successes and that the whole Chinese system can be dispensed with. There is no reason, however, why evaluation of traditional Chinese medicine needs to be this holistic. Some pre-scientific medical practices, such as the North American aboriginals chewing salicin-containing willow bark to relieve pain, have turned out to be medically effective even by modern standards. It is entirely possible, therefore, that some traditional Chinese therapies such as acupuncture and herbal remedies might have some efficacy.

Acupuncture is a family of procedures, the most familiar of which involves penetration of specific points on the skin by thin metallic needles. If acupuncture were only comprehensible within traditional Chinese medicine, then it might indeed be concluded that acupuncture is strongly incommensurable with the substantially different system of Western medicine. But acupuncture has in fact been evaluated from the perspective of Western medicine, most recently and publicly by the NIH Consensus Development Conference that took place in November, 1997. The operations of this conference are a striking example of evaluation occurring in the face of conceptual difficulties. Acupuncture would never have been invented within Western medical science, but that does not make it immune to scientific evaluation.

Like previous NIH Consensus Conferences, the acupuncture conference consisted of one and a half days of presentations followed the next morning by presentation of a consensus report. This report was prepared by a 12-member panel drawn from different 
backgrounds, including both acupuncture specialists and Western-trained medical experts. Panel members worked until 4 a.m. on the final day of the conference to reach agreement on a statement that was publicly released later that morning. ${ }^{3}$ The panel concluded that "there is clear evidence that needle acupuncture is efficacious for adult post-operative and chemotherapy nausea and vomiting and probably for the nausea of pregnancy." It also found some evidence of efficacy for postoperative dental pain, and suggestive but not conclusive evidence for pain relief in other conditions such as menstrual cramps. Since acupuncture has minimal adverse effects, the panel stated that acupuncture may be a reasonable option for a number of clinical conditions such as stroke rehabilitation and osteoarthritis.

The panel reached its conclusions using the standards of Western medicine. Ideally, evaluation of medical effectiveness should be based on randomized, controlled, blinded clinical trials, but such trials have only been a part of medical research since World War II, and most Western medical practices are based on medical experience rather than rigorous tests. With a procedure as obvious as acupuncture, it is not easy to perform properly controlled experiments: unlike placebo pills, patients clearly know whether they have received acupuncture or not. Experiments using "sham" acupuncture, in which needles are inserted at non-standard acupuncture points, have provided mixed results often intermediate between orthodox acupuncture and nontreatment. The panel decided not to insist on the highest standards of medical efficacy based only on rigorously controlled experiments, but rather to evaluate acupuncture based on the more usual clinical standards of Western medicine. The panel concluded that acupuncture may well be effective for the treatment of nausea and pain, and recommended future high-quality, randomized, controlled clinical trials on its effects.

The panel's recommendations were based on a large body of printed information provided by NIH in advance of the conference, and on the presentations of twenty-four speakers on the first day and a half of the conference. Although a few of the talks 
presented acupuncture within the context of traditional Chinese medicine, the vast majority discussed its effectiveness from the Western evidential perspective. Several talks discussed the possible neurochemical basis of acupuncture, presenting evidence that acupuncture stimulates the production of endogenous opioids and affects the secretion of neurotransmitters and neurohormones. The panel report, however, remained open to the traditional Chinese medicine based on qi: "Although biochemical and physiologic studies have provided insight into some of the biologic effects of acupuncture, acupuncture practice is based on a very different model of energy balance. This theory may provide new insights to medical research that may further elucidate the basis for acupuncture." This statement is not an endorsement of traditional Chinese medicine, but it suggests that its theory as well as its practice may turn out to be useful in Western scientific medicine. The implication, however, is that the theory of $q i$ would need to be evaluated according to scientific standards, not in accord with traditional Chinese texts or the doctrine of correspondences.

According to some sociologists, science is essentially a power play in which some researchers marshal resources to triumph over others (Latour 1987). One interpretation of the NIH consensus conference would be that acupuncture proponents managed to dominate by assembling speakers and panel members to endorse their claims. Alternatively, the conference organizers could conceivably have assembled a panel of hard-line Western medical researchers who would have dismissed acupuncture as pseudoscientific trickery. Although consensus conferences undoubtedly have a political dimension, their operation is designed to encourage evidence evaluation rather than political manipulation. The twelve panel members were presented with a common body of information to evaluate, and most of them had no strong interest for or against acupuncture; only two of the twelve were practicing acupuncturists, and both had Western medical training. Some of the studies they looked at, particularly the well-done 
and replicated studies concerning the effects of acupuncture on post-operative nausea, were very impressive.

\section{Conceptual Change as Intentional and Emotional}

We have argued that the substantial conceptual differences between traditional Chinese medicine and Western medicine can be overcome, but it would be rash to exaggerate the ease with which mutual understanding can be accomplished. Consider two people, one an expert on and a proponent of traditional Chinese medicine - $\mathrm{C}-$ and the other trained in Western medicine - W. Initially, C and W will scarcely be able to talk to each other, with the former using concepts like $q i$ and the other using concepts like germ and immune system. Any degree of mutual comprehension that develops will depend not only on casual communication, which will be ineffective, but on the kind of intentional conceptual change that is discussed in this volume. $\mathrm{C}$ and $\mathrm{W}$ each must have the motivation to acquire enough of the other's conceptual system that comparison and evaluation becomes possible. Only then do $\mathrm{C}$ and $\mathrm{W}$ have the capability of changing their conceptual systems by adopting components of the alternative system and by revising their own concepts (see Thagard 1992 for a taxonomy of conceptual changes).

People who undergo conceptual change, whether from the traditional Chinese system of medicine to the Western system or vice versa, must have a set of cognitive goals that directs their thinking. First, they must have the goal of understanding the alternative system, which requires becoming familiar with (but not necessarily endorsing) its concepts, hypotheses, and evidence. Accomplishing this goal may involve trying to translate the alternative system into more familiar terms, or of understanding the system on its own terms. Second, they must have the goal of assessing the alternative systems with respect to explanatory coherence and practical efficacy. Third, they must be willing to recognize an alternative conceptual system as superior in important respects to their own and therefore worthy of replacing it, partially or totally. Thus the development of mutual understanding and the process of conceptual change depend in part of the 
intentions of people to take seriously conceptual systems that differ from the ones they currently hold.

One major impediment to conceptual change that has been largely ignored in psychological and philosophical discussions is the emotional attachment that people have to their own systems. Like all thinking, scientific cognition is in part an emotional process (Thagard forthcoming). People do not only hold and use their concepts and hypotheses, they also feel emotionally attached to them and respond with negative emotions to concepts and hypotheses that clash with them. For a proponent of traditional Chinese medicine, acupuncture may be a revered practice associated with happy outcomes, whereas for a Western physician it may seem like a ridiculous throwback to prescientific practices held in contempt. Conceptual change about different approaches to medicine involves changing not only concepts, hypotheses, and practices but also emotional attitudes toward those concepts, hypotheses, and practices. Having the intention to understand and evaluate alternative views can make the emotional component of conceptual change more easily realized.

\section{Conclusion}

We embarked on this study in order to examine a more extreme case of possible incommensurability than typically occurs in the history of Western science. The issue is philosophically important because questions about incommensurability raised by Kuhn and Feyerabend are often used to support relativist views that challenge the rationality of science (Laudan 1990). Our examination has shown that there are indeed linguistic, conceptual, ontological, and explanatory differences that make mutual evaluation of traditional Chinese medicine and Western scientific medicine difficult. We have also seen, however, that these difficulties can to a great extent be overcome by earnest, intentional attempts to learn alternative languages, conceptual schemes, and explanatory patterns. As the NIH consensus conferences shows, a therapeutic practice like acupuncture can be evaluated for its effectiveness without adopting the theoretical 
framework from which it arose. We do not need to have a grand, holistic clash of traditional Chinese medicine versus Western scientific medicine to conduct a useful piecemeal evaluation of particular treatments. The two systems of medicine are weakly incommensurable (mutually untranslatable), but they are not strongly incommensurable (mutually unintelligible). Despite the substantial barriers to complete translation that divide different systems of medicine, rational scientific evaluation of practices such as acupuncture is possible.

\section{References}

Allchin, D. (1996). Points east and west: Acupuncture and comparative philosophy of science. Philosophy of Science, 63(supplement), S107-S115.

Au, T. K. (1983). Chinese and English counterfactuals: The Sapir-Whorf hypothesis revisited. Cognition, 15, 155-187.

Bloom, A. (1981). The linguistic shaping of thought: A study of the impact of language on thinking in China and the West. Hillsdale, NJ: Erlbaum.

Cheng, P. W. (1985). Pictures of ghosts: A critique of Alfred Bloom's The Linguistic Shaping of Thought. American Anthropologist, 87, 917-922.

Darden, L., \& Cain, J. (1989). Selection type theories. Philosophy of Science, 56, 106129.

Feyerabend, P. K. (1981). Realism, rationalism, and scientific method. Cambridge: Cambridge University Press.

Giere, R. N. (1994). The cognitive structure of scientific theories. Philosophy of Science, 61, 276-296.

Guo, A. (Ed.). (1992). Huang di nei jing su wen jiao zhu (Annotations on Yellow Emperor's classic of internal medicine: Plain questions, in Chinese). Beijing: People's Medical Press. 
Kelley, H. H. (1972). Causal schemata and the attribution process. In E. E. Jones, D. E. Kanouse, H. H. Kelley, R. E. Nisbett, S. Valins, \& B. Weiner (Eds.), Attribution: Perceiving the causes of behavior. Morristown NJ: General Learning Press.

Kitcher, P. (1981). Explanatory unification. Philosophy of Science, 48, 507-531.

Kitcher, P. (1989). Explanatory unification and the causal structure of the world. In P. Kitcher \& W. C. Salmon (Eds.), Scientific explanation (pp. 410-505). Minneapolis: University of Minnesota Press.

Kitcher, P. (1993). The advancement of science. Oxford: Oxford University Press.

Kuhn, T. (1970). Structure of scientific revolutions. (2 ed.). Chicago: University of Chicago Press.

Kuhn, T. S. (1993). Afterwords. In P. Horwich (Ed.), World changes: Thomas Kuhn and the nature of science (pp. 311-341). Cambridge, MA: MIT Press.

Latour, B. (1987). Science in action: How to follow scientists and engineers through society. Cambridge, MA: Harvard University Press.

Laudan, L. (1984). Science and values. Berkeley: University of California Press.

Laudan, L. (1990). Science and relativism. Chicago: University of Chicago Press.

Leake, D. B. (1992). Evaluating explanations: A content theory. Hillsdale, NJ: Erlbaum.

Lewis, D. (1986). Philosophical papers. Oxford: Oxford University Press.

Lloyd, G. E. R. (1996). Adversaries and authorities. Cambridge: Cambridge University Press.

Lu, G., \& Needham, J. (1980). Celestial lancets: A history and rationale of acupuncture and moxa. Cambridge: Cambridge University Press.

Moyers, B. (1993). Healing and the mind. New York: Doubleday.

Nersessian, N. (1984). Faraday to Einstein: Constructing meaning in scientific theories. Dordrecht: Martinus Nijhoff.

Porkert, M., \& Ullmann, C. (1988). Chinese medicine (Howson, M., Trans.). (1st U.S. -ed.). New York: Morrow. 
Sackett, D. L. e. a. (1996). Evidence-based medicine: What it is and what it isn't. British Medical Journal, 312, 71-72.

Schaffner, K. F. (1993). Discovery and explanation in biology and medicine. Chicago: University of Chicago Press.

Schank, R. C. (1986). Explanation patterns: Understanding mechanically and creatively. Hillsdale, NJ: Erlbaum.

Shen, Z., \& Chen, Z. (1996). The basis of traditional Chinese medicine. Boston: Shambhala.

Thagard, P. (1992). Conceptual revolutions. Princeton: Princeton University Press.

Thagard, P. (1996). The concept of disease: Structure and change. Communication and Cognition, 29, 445-478.

Thagard, P. (1998). Explaining disease: Causes, correlations, and mechanisms. Minds and Machines, 8, 61-78.

Thagard, P. (1999). How scientists explain disease. Princeton, NJ: Princeton University Press.

Thagard, P. (forthcoming). The passionate scientist: Emotions in scientific cognition. In P. Carruthers (Ed.), The cognitive basis of science .

Unschuld, P. U. (1985). Medicine in China: A history of ideas. Berkeley: University of California Press.

Whorf, B. (1956). Language, thought, and reality. Cambridge, MA: MIT Press.

Zhen, Z. (Ed.). (1997). Zhong guo yi xue shi (History of Chinese medicine, in Chinese) (2nd ed.). Shanghai: Shanghai Science and Technology Press. 
${ }^{1}$ We are grateful to Daniel Moerman and Cameron Shelley for comments on an earlier draft, and to the Social Sciences and Humanities Research Council of Canada for financial support.

${ }^{2}$ Explanation schemas and similar abstractions have been discussed in philosophy and cognitive science using varying terminology. See e.g. Darden and Cain (1989), Giere (1994), Kelley (1972), Kitcher (1981, 1989, 1993), Leake (1992), Schaffner (1993), Schank (1986), and Thagard (1988, 1992b).

${ }^{3}$ The consensus statement and press release, as well as the abstracts of presentations, are available on the World Wide Web at http://odp.od.nih.gov/consensus/statements/cdc/107/107_intro.html. The statement will eventually be published in a medical journal. We are indebted to one of the panel members, Daniel Moerman, for information on the panel deliberations. 